\title{
D-Pinitol alleviates cyclosporine A-induced renal tubulointerstitial fibrosis via activating Sirt1 and Nrf2 antioxidant pathways
}

\author{
EUN SIL KOH ${ }^{1}$, SOOJEONG KIM ${ }^{2}$, MINYOUNG KIM ${ }^{1}$, YU AH HONG ${ }^{1}$, SEOK JOON SHIN ${ }^{1}$, \\ CHEOL WHEE PARK ${ }^{1}$, YOON SIK CHANG ${ }^{1}$, SUNGJIN CHUNG ${ }^{1,3}$ and HO-SHIK KIM ${ }^{2}$ \\ ${ }^{1}$ Department of Internal Medicine, College of Medicine, The Catholic University of Korea; ${ }^{2}$ Department of Biochemistry, \\ College of Medicine, The Catholic University of Korea, Seoul 06591, Republic of Korea; ${ }^{3}$ Division of Nephrology \\ and Hypertension, Department of Medicine, School of Medicine, Vanderbilt University, Nashville, TN 37232-2372, USA
}

Received July 7, 2017; Accepted January 2, 2018

DOI: $10.3892 /$ ijmm.2018.3408

\begin{abstract}
Although the mechanism of cyclosporine A (CsA)-induced renal injury remains to be fully elucidated, accumulating evidence suggests that oxidative stress is critical in producing CsA-induced structural and functional renal impairment. The present study investigated the effect of D-pinitol, a cyclitol present in soybean, on chronic CsA nephropathy. Male ICR mice were treated with vehicle, CsA (30 mg/kg/day), D-pinitol (50 mg/kg/day) or a combination of CsA and D-pinitol for 28 days. To assess which pathway responding to oxidative stress is augmented by D-pinitol, the expression levels of several antioxidant enzymes and their possible regulators were measured. Treatment with D-pinitol significantly suppressed the increase of serum creatinine and decrease of urine osmolality, compared with the CsA control group. Histological examination of Masson's trichrome- and $\alpha$-smooth muscle actin-stained renal tissue demonstrated that the CsA-induced tubulointerstitial fibrosis and inflammation were attenuated by D-pinitol. Following the administration of D-pinitol, there were increased expression levels of heme oxygenase-1, NAD(P)H:quinone oxidoreductase 1, superoxide dismutase 1 and catalase in CsA-treated kidneys. In addition, D-pinitol increased the level of sirtuin 1 (Sirt1), and the total and nuclear expression levels of nuclear erythroid factor 2-related factor 2 (Nrf2), suggesting that activation of the Sirt1 and Nrf2 pathways may induce the cellular antioxi-
\end{abstract}

Correspondence to: Professor Sungjin Chung, Department of Internal Medicine, College of Medicine, The Catholic University of Korea, 10, 63-ro, Yeongdeungpo-gu, Seoul 06591, Republic of Korea E-mail: chungs@catholic.ac.kr

Professor Ho-Shik Kim, Department of Biochemistry, College of Medicine, The Catholic University of Korea, 222 Banpo-daero, Seocho-gu, Seoul 06591, Republic of Korea

E-mail: hoshik@catholic.ac.kr

Key words: D-pinitol, cyclosporine, fibrosis, nuclear erythroid factor 2-related factor 2, sirtuin 1 dant system against CsA-induced nephropathy. Collectively, these data suggested that D-pinitol may protect the kidney from CsA-induced fibrosis, and that this renoprotective effect of D-pinitol was due to the inhibition of oxidative stress through the activation of Sirt1 and Nrf2, and the subsequent enhancement of antioxidant enzymes.

\section{Introduction}

D-pinitol, the 3-O-methyl form of D-chiro-inositol, which is abundant in Pinaceae and Leguminosae plants is a natural inositol derivative functioning as an active principle in soy foods and legumes $(1,2)$. D-pinitol has been shown to possess multifunctional properties, including antidiabetic, antilipidemic and anticancer effects (2-5). As one of the potential mechanisms for its anti-diabetic effect, it has been reported that D-pinitol increases insulin-mediated glucose uptake in the liver by activating the phosphatidylinositol-3-kinase (PI3K)/Akt signalling pathway in a type 2 diabetic experimental rat model (2). It has been also demonstrated that D-pinitol improves insulin sensitivity by stimulating the translocation of glucose transporter type 4 (GLUT4) to the plasma membrane in the skeletal muscle of diabetic mice (6). Consistent with the results in the diabetic animal model, D-pinitol supplementation improves glycemic control in healthy control and diabetic patients, although there are conflicting results $(7,8)$. In addition to its attenuating effect on insulin resistance, D-pinitol appears to ameliorate hyperlipidemia, inflammation and oxidative stress in the liver, kidney and pancreas in diabetic animals. Sivakumar et al (9) demonstrated that D-pinitol protects the kidney by attenuating hyperglycemia-induced proinflammatory cytokines and oxidative stress, resulting in histological and ultra-structural improvement in streptozotocin-induced diabetic rats. This indicates that the anti-oxidative effect of D-pinitol may be a pivotal factor in its renoprotective effect against diabetes.

The introduction of cyclosporine A (CsA) revolutionized solid organ transplantation, reducing rejection rates and improving early graft survival (10). Although there is a trend in introducing novel immunosuppressive agents, CsA remains the backbone of current immunosuppression for kidney transplantation. In addition, accumulating evidence has shown that CsA is effective in a significant number of patients with 
steroid-resistant nephrotic syndrome (10), and it has also been used to treat frequently relapsing nephrotic syndrome (11). Despite its significant contributions to transplantation and treatment of proteinuric renal diseases, patients treated with CsA can suffer from a range of side effects, including nephrotoxicity (12). The acute effects of CsA present mainly as vasoconstriction of the afferent arteriole, which can occur even following administration of the first dose, and CsA can also cause renal morphological changes known as chronic CsA nephropathy (13). Although the exact mechanism of CsA-induced renal injury remains to be fully elucidated, evidence has demonstrated that CsA-induced oxidative stress is critical in causing structural and functional impairment of the kidney (14-16), This suggests that the attenuation of oxidative stress has a protective effect in kidneys exposed to CsA. Our previous study demonstrated that oleanolic acid, a natural pentacyclic triterpenoid, had a renoprotective effect by decreasing oxidative stress generated by CsA (17).

The present study aimed to identify whether D-pinitol has a protective effect against CsA-induced nephropathy and to analyze the underlying mechanism with a focus on the anti-oxidative effect of D-pinitol.

\section{Materials and methods}

Experimental design. The present study was approved by The Institutional Animal Care and Use Committee of The Catholic University of Korea Yeouido St. Mary's Hospital (Seoul, Korea; approval no. YEO20131602FA). Five-week-old male ICR mice (DooYeol Biotech., Seoul, Korea) with initial weights of $15-20 \mathrm{~g}$ were housed at room temperature $\left(22 \pm 1^{\circ} \mathrm{C}\right)$ under an alternating $12 \mathrm{~h}$-light and $12 \mathrm{~h}$-dark cycle. The animals were provided with free access to a low-salt diet (0.01\% sodium; Research Diets, Inc., New Brunswick, NJ, USA) and water ad libitum. The mice were allowed to acclimatize for 1 week prior to experiments. The mice were assigned into four groups: i) Vehicle-treated control group (control, $n=8$ ), in which mice were administered with a daily subcutaneous injection of vehicle ( $1 \mathrm{ml} / \mathrm{kg}$ olive oil; Merck Millipore, Darmstadt, Germany); ii) vehicle and D-pinitol-treated group (pinitol, $\mathrm{n}=8)$, in which mice were administered with a daily subcutaneous injection of olive oil (1 ml/ $\mathrm{kg}$ ) and D-pinitol $(50 \mathrm{mg} / \mathrm{kg}$; Merck Millipore) daily; iii) CsA only group (CsA, n=8), in which mice were administered with a daily subcutaneous injection of CsA (30 mg/kg; Chong Kun Dang Pharmaceutical Corp., Seoul, Korea); iv) CsA and D-pinitol-treated group (CsA+pinitol, $\mathrm{n}=8$ ), in which mice were administered with a daily subcutaneous injection of CsA (30 mg/kg) and D-pinitol (50 mg/kg) orally for 4 weeks. The doses of CsA and D-pinitol were selected based on a previous study (6).

Assessment of basic parameters of renalfunction. Twenty-fourhour urine collection was performed using metabolic cages (Nalgene, Rochester, NY, USA) prior to sacrifice. Proteinuria and urine creatinine levels were measured using ELISA kits (Exocell, Inc., Philadelphia, PA, USA). Measurement of serum creatinine concentration and urine osmolality was performed at Samkwang Medical Laboratories (Seoul, Korea) using enzymatic colorimetric methods (Modular DPP system; Roche Diagnostics GmbH, Hamburg, Germany). Creatinine clearance was calculated using a standard formula: Urine creatinine $(\mathrm{mg} / \mathrm{dl}) \times$ urine volume $(\mathrm{ml} / 24 \mathrm{~h}) /$ serum creatinine (mg/dl) x1440 (min/24 h). Whole-blood CsA concentration was determined by liquid chromatography-tandem mass spectrometry using an API3200 LC-MS system (MDS Sciex, Foster City, CA, USA) equipped with an electrospray ionization interface to generate negative ions $[\mathrm{M}+\mathrm{NH} 4]^{+}$.

Histopathology. On the day of sacrifice, the kidneys were retrieved, washed with heparinized saline, fixed in a periodate-lysine-paraformaldehyde solution and embedded in wax. Following dewaxing, sections with a thickness of 4- $\mu \mathrm{m}$ were processed and stained with Masson's trichrome. Tubulointerstitial fibrosis was defined as a matrix-rich expansion of the interstitium with tubular dilatation, atrophy, cast formation, and sloughing of tubular epithelial cells or thickening of the tubular basement membrane. At least 20 fields per section were assessed by counting the percentage of injured area per field of cortex at 200x magnification using a color-image analyzer (TDI Scope Eye Version 3.5 for Windows; Olympus, Tokyo, Japan).

Immunohistochemistry. For immunohistochemistry, the 4- $\mu$ m-thick sections were deparaffinized, hydrated in ethanol and treated with an antigen unmasking solution containing $10 \mathrm{mM}$ sodium citrate buffer ( $\mathrm{pH}$ 6.0) followed by washing with PBS. The sections were incubated with $3 \% \mathrm{H}_{2} \mathrm{O}_{2}$ in methanol to block endogenous peroxidase activity. Non-specific binding was blocked with $10 \%$ normal goat serum in PBS. The sections were incubated with anti- $\alpha$-smooth muscle actin ( $\alpha$-SMA; dilution, 1:1,000; cat. no. ab32575) or anti-collagen IV (dilution, 1:1,000; cat. no. ab6586) (both from Abcam, Cambridge, UK) antibodies overnight in a humidified chamber at $4^{\circ} \mathrm{C}$. Antibodies were then localized through incubation with a peroxidase-conjugated horse anti-rabbit IgG (dilution, ready to use) for $1 \mathrm{~h}$ at room temperature and DAB substrate solution using the Vector Immpress kit (cat. no. MP-7401; Vector Laboratories, Inc., Burlingame, CA, USA). The sections were dehydrated in ethanol, cleared in xylene and mounted without counterstaining. The sections were examined in a blinded-manner using light microscopy (Olympus BX-50; Olympus). For quantification of the proportional area of staining, 20 views (magnification, $\mathrm{x} 400$ ) were randomly located in the renal cortex and cortico-medullary junction of each slide. Images were captured and analyzed to determine density x positive area/glomerular total area using Image J software 1.49 (National Institutes of Health, Bethesda, MD, USA).

Immunoblot analysis. Immunoblot analysis was performed to analyze the effect of D-pinitol on the expression of several important proteins in the pathogenesis of UUO-induced renal fibrosis. Whole cell lysates and nuclear fractions of the renal cortical tissues were extracted using Pro-Prep protein extraction solution (Intron Biotechnology, Inc., Seongnam, Korea) and the NE-PER nuclear kit (Pierce; Thermo Fisher Scientific, Inc., Waltham, MA, USA), respectively, according the manufacturer's protocol (18). Protein concentration of whole cell lysates and nuclear fractions were determined using BCA assay kit (Thermo Fisher Scientific, Inc.). Equal 
Table I. Physical and biochemical characteristics of the four groups at the end of the 4-week period.

\begin{tabular}{lcccc}
\hline Characteristic & Control $(\mathrm{n}=8)$ & Pinitol $(\mathrm{n}=8)$ & CsA $(\mathrm{n}=8)$ & CsA+pinitol $(\mathrm{n}=8)$ \\
\hline Body weight $(\mathrm{g})$ & $33.68 \pm 1.73$ & $32.94 \pm 0.90$ & $29.81 \pm 1.19$ & $28.88 \pm 2.45$ \\
Urine volume $(\mathrm{ml})$ & $2.16 \pm 2.39$ & $2.03 \pm 1.88$ & $5.25 \pm 1.44^{\mathrm{a}}$ & $6.26 \pm 3.20^{\mathrm{b}}$ \\
Serum Cr $(\mathrm{mg} / \mathrm{dl})$ & $0.11 \pm 0.02$ & $0.13 \pm 0.02$ & $0.27 \pm 0.11^{\mathrm{c}}$ & $0.17 \pm 0.16^{\mathrm{d}}$ \\
Urine osmolality $(\mathrm{mOsm} / \mathrm{kg})$ & $1324.90 \pm 534.50$ & $1280.00 \pm 230.20$ & $494.00 \pm 208.80^{\mathrm{e}}$ & $705.80 \pm 280.00$ \\
Cr clearance $(\mathrm{ml} / \mathrm{min} / 100 \mathrm{~g} \mathrm{BW})$ & $1.29 \pm 0.15$ & $1.38 \pm 0.09$ & $0.52 \pm 0.16^{\mathrm{f}}$ & $1.03 \pm 0.19$ \\
Urine protein/Cr ratio $(\mathrm{mg} / \mathrm{mg})$ & $23.30 \pm 4.48$ & $17.80 \pm 11.88$ & $8.40 \pm 5.70^{\mathrm{g}}$ & $3.80 \pm 2.00$ \\
CsA concentration $(\mathrm{ng} / \mathrm{ml})$ & N/A & N/A & $896.20 \pm 328.40$ & $882.20 \pm 302.90$ \\
\hline
\end{tabular}

Data are presented as the mean \pm standard error of the mean. ${ }^{\mathrm{a}} \mathrm{P}=0.013$ vs. control group; ${ }^{\mathrm{b}} \mathrm{P}=0.009$ vs. control group; ${ }^{\mathrm{c}} \mathrm{P}=0.001 \mathrm{vs}$. control group; ${ }^{\mathrm{d}} \mathrm{P}=0.042$ vs. CsA group; ${ }^{\mathrm{e}} \mathrm{P}=0.005$ vs. control group; ${ }^{\mathrm{f}} \mathrm{P}=0.028$ vs. control group; ${ }^{\mathrm{g}} \mathrm{P}=0.001$ vs. control group. Cr, creatinine; $\mathrm{CsA}$, cyclosporine-A; control, vehicle-treated control group; Pinitol, D-pinitol; N/A, not available.

amount $(20 \mu \mathrm{g})$ of each sample was then separated by $12 \%$ SDS-PAGE, and transferred onto nitrocellulose membranes. Subsequent to blocking in $3 \%$ bovine serum albumin in TBS-T solution for $1 \mathrm{~h}$ at room temperature, the membranes were incubated with primary antibodies overnight at $4^{\circ} \mathrm{C}$. Following incubation with secondary antibodies conjugated with horseradish peroxidase for $1 \mathrm{~h}$, at room temperature, the protein was visualized using an enhanced chemiluminescence detection method (GE Healthcare Life Sciences, Chalfont, UK). Primary antibodies against nuclear erythroid factor 2-related factor 2 (Nrf2; 1:1,000; cat. no. sc722), Kelch-like ech-associated protein 1 (Keap1; 1:1,000; cat. no. sc33569), NAD(P)H:quinone oxidoreductase 1 (NQO1; 1:1.000; cat. no. sc16464) and acetylated Forkhead box O1 (FoxO1; 1:1,000; cat. no. sc49437) were purchased from Santa Cruz Biotechnology, Inc. (Dallas, TX, USA), and antibodies against Sirt1 (1:1,000; cat. no. 9475), Akt (1:1,000; cat. no. 9272), phospho-Akt (1:1,000; cat. no. 9271) and lamin B1 (1:1,000; cat. no. 125860) were from Cell Signaling Technology, Inc (Danvers, MA, USA). Antibodies against superoxide dismutase SOD1 (SOD1; 1:10,000; cat. no. ADI-SOD-100; Enzo Life Sciences, Inc., Farmingdale, NY, USA), SOD2 (dilution, 1:10,000; cat. no. ab16956), catalase (dilution, 1:2,000; cat. no. ab31630), FoxO1 (dilution, 1:1,000; cat. no. ab39670), phospho-FoxO1A S329 (dilution, 1:1,000; cat. no. ab58519) (all from Abcam), heme oxygenase-1 (HO-1; dilution, 1:1,000; cat. no. PA5-27338; BD Biosciences, Franklin Lakes, NJ, USA), and $\beta$-actin (dilution, 1:10,000; cat. no. 061M4808; Merck Millipore) were also commercially obtained.

Terminal deoxynucleotidyltransferase-mediated biotin nick end-labeling (TUNEL) assay. Apoptosis was assessed using TUNEL assay. Apoptotic cells in the formalin-fixed and paraffin-embedded tissue were detected using the ApopTag In Situ Apoptosis Detection kit (EMD Millipore, Billerica, MA, USA) according to the manufacturer's protocol. TUNEL-positive cells were evaluated under a light microscope (Olympus BX-50; Olympus) at 400x magnification.

Statistical analysis. Data are expressed as the mean \pm standard error of the mean. Differences between groups were examined for statistical significance using one-way analysis of variance with Bonferroni correction using SPSS version 19.0 (IBM SPSS, Armonk, NY, USA). P<0.05 was considered to indicate a statistically significant difference. Each experiment was independently performed at least three times.

\section{Results}

Effect of D-pinitol on renal functional changes. Following 28 days of treatment, the parameters of renal functions were measured as summarized in Table I. The 24-h urine volume was significantly increased in the CsA group and the CsA+pinitol group. The CsA-treated mice had significantly lower urinary osmolality. The serum creatinine level was significantly increased and creatinine clearance was decreased in the CsA group. However, D-pinitol treatment enhanced creatinine clearance by reducing the CsA-induced elevation of serum creatinine, and increased urine osmolality (Table I).

Effect of D-pinitol on renal morphological changes. To evaluate whether D-pinitol affected renal morphological changes, staining with Masson-trichrome, $\alpha$-SMA, and collagen IV was performed. The tubulointerstitial fibrosis, analyzed using Masson-trichrome staining, was increased in the kidney tissues of the CsA-treated mice, compared with that in the control group. By contrast, D-pinitol treatment significantly attenuated renal fibrosis in the CsA-treated mice (Fig. 1A and B). CsA treatment also led to the significant upregulation of $\alpha$-SMA, a marker of myofibroblasts, and type IV collagen in the kidneys of the mice. By contrast, D-pinitol mitigated the CsA-induced expression of $\alpha$-SMA and type IV collagen (Fig. 1A, C and D).

Effect of D-pinitol on renal oxidative status. Following 28 days of CsA administration, there was a significant decrease in renal SOD1 (Fig. 2A and B). D-pinitol treatment significantly ameliorated the CsA-induced reduction in SOD1. By contrast, no significant difference was found in the expression of SOD2 among the groups (Fig. 2A and C). The renal levels of HO-1, NQO-1 and catalase were also determined. It was found that D-pinitol treatment of the CsA-treated mice restored the decreased expression of HO-1 in the kidney (Fig. 3A and B). There was increased expression of NQO1 in the CsA-treated mice, compared with that in the control, which was more 

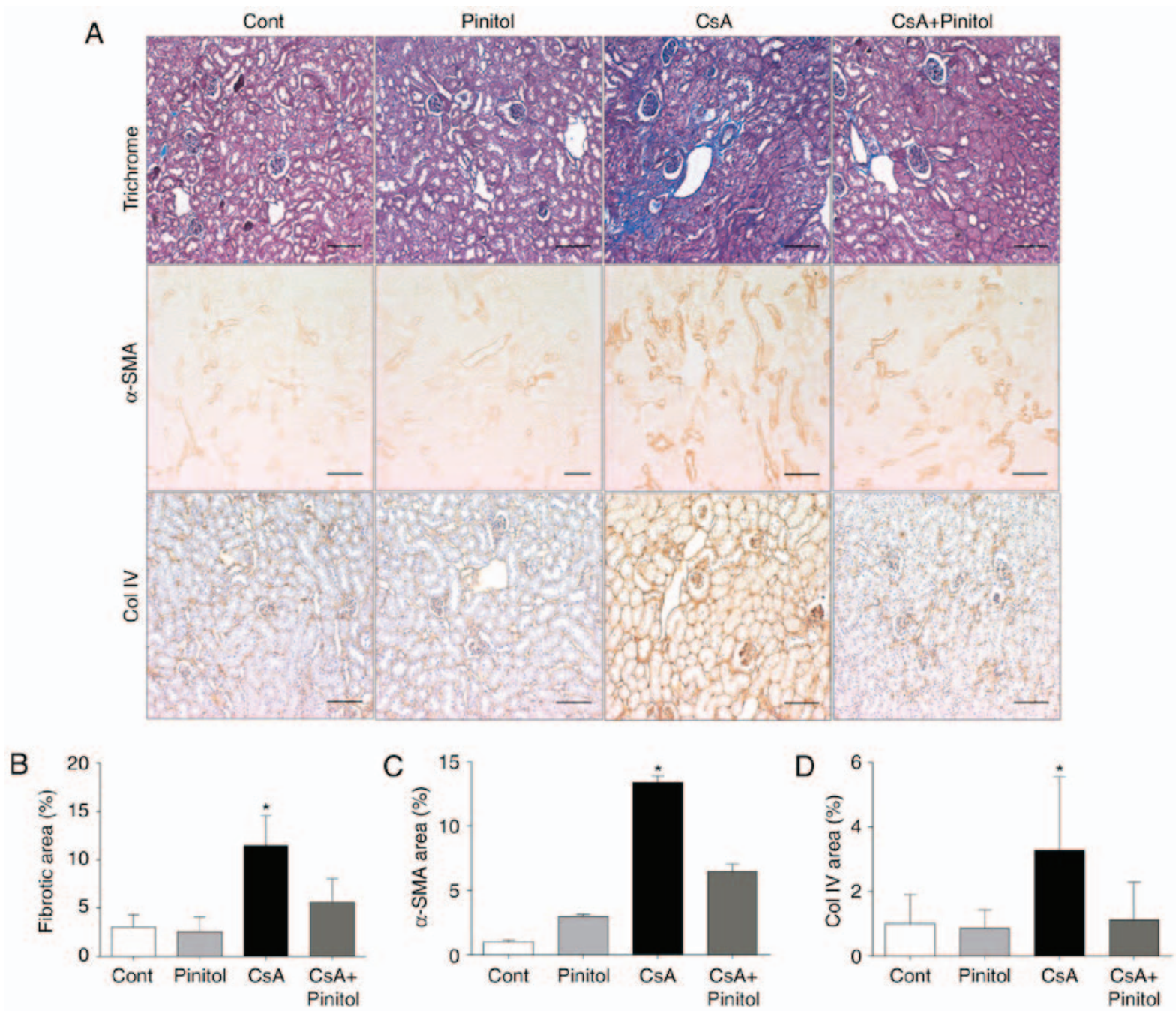

Figure 1. Effect of D-pinitol on CsA-induced renal morphological changes. (A) Representative renal sections stained with Masson-trichrome, $\alpha$-SMA and Col IV (original magnification, x200). Scale bar, $2 \mu \mathrm{m}$. (B) Quantitative analyses of results for tubulointerstitial fibrosis area. "P $<0.001$ vs. other groups. (C) Quantitative analyses of results for $\alpha$-SMA. " $\mathrm{P}<0.001$ vs. other groups. (D) Quantitative analyses of results for collagen IV staining. "P<0.001 vs. other groups. Trichrome, Masson's trichrome; $\alpha$-SMA, $\alpha$-smooth muscle actin; Col IV, collagen IV; Cont, vehicle-treated control group; Pinitol, D-pinitol; CsA, cyclosporine.

marked following D-pinitol treatment (Fig. 3A and C). D-pinitol treatment restored the decreased expression of catalase in the CsA-treated mice (Fig. 3A and D). Taken together, the intrarenal levels of cytosolic antioxidant enzymes were increased by D-pinitol treatment, suggesting that the increased activity of cytosolic antioxidant enzymes may be attributed to the renoprotective effects of D-pinitol against CsA-induced nephropathy.

Effect of D-pinitol on the Nrf2 signalling pathway. It has been reported that HO-1, catalase and SOD1 are major transcriptional target genes of Nrf2 (19). Accordingly, it was necessary to determine whether D-pinitol treatment affected the Nrf2/Keap1 signalling pathway. The results of the western blot analysis showed that CsA markedly decreased the nuclear expression of Nrf2, whereas the expression levels of Keap1 and total Nrf2 were marginally increased in the CsA mice (Fig. 4A-C). It was noted that D-pinitol treatment restored the nuclear expression of Nrf2 in the kidneys of CsA-treated mice (Fig. 4A and D).

Effect of D-pinitol on the renal expression of Sirt1, Akt and FoxOl. Sirt1 and its downstream pathway are crucial in several biological pathways, including apoptosis and oxidative stress responses in chronic kidney disease (20). Therefore, the effect of D-pinitol treatment on Sirt1 and its downstream pathway was determined. The results of the western blot analysis revealed that the intrarenal expression level of Sirt1 was increased in the CsA+pinitol group, compared with that in the other groups (Fig. 5A and B). By contrast, the ratios of phospho-Akt/Akt, acetylated FoxO1/FoxO1 and phospho-FoxO1/FoxO1 were all increased in the kidneys of the CsA-treated mice (Fig. 6). These ratios were significantly decreased following treatment with D-pinitol.

Effect of D-pinitol on renal apoptosis. To evaluate whether D-pinitol treatment affected intrarenal apoptosis, TUNEL assay was performed. It was found that the number of TUNEL-positive cells in the renal interstitium of the CsA-treated mice was significantly increased, compared with that in the control group. This was reversed by D-pinitol treatment (Fig. 7), indicating that D-pinitol treatment attenuated apoptosis in CsA nephropathy. 

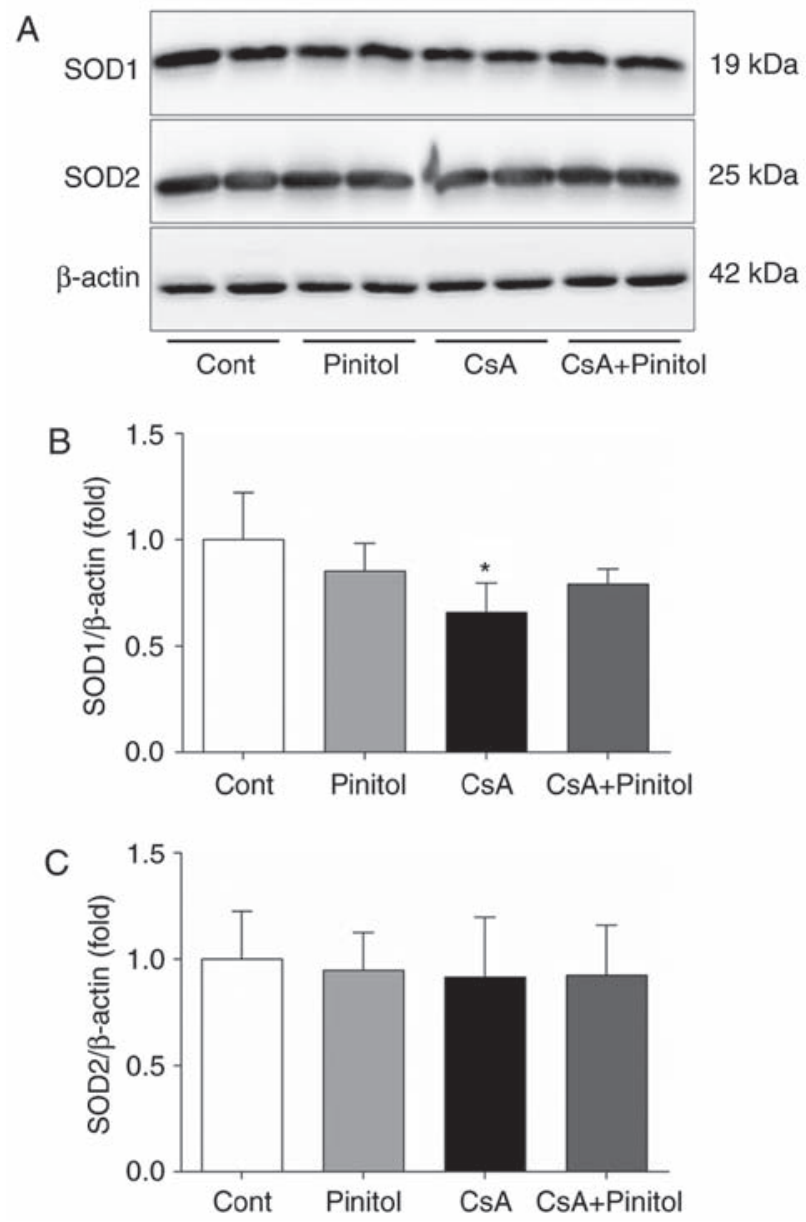

Figure 2. Effect of D-pinitol on renal expression of SODs in chronic CsA nephropathy. (A) Representative western blot showing the effect of D-pinitol on the expression of SOD1 and SOD2 in chronic CsA nephropathy. (B) Quantitative analyses for $\mathrm{SOD} 1 / \beta$-actin. ${ }^{*} \mathrm{P}=0.050$ vs. $\mathrm{CsA}+$ pinitol group. (C) Quantitative analyses for SOD2/ $\beta$-actin. Cont, vehicle-treated control group; Pinitol, D-pinitol; CsA, cyclosporine A; SOD, superoxide dismutase.

\section{Discussion}

In the present study, it was demonstrated that D-pinitol treatment recovered renal function in a mouse model of chronic CsA nephropathy. This renoprotective effect of D-pinitol was associated with improvement in renal fibrosis, apoptotic cell injury and oxidative stress through restoration of the decreased Nrf2 and Sirt1 pathway. It was shown that Nrf2 and its downstream genes, including SOD1, HO-1, NQO1 and catalase, were all increased by D-pinitol. Additionally, D-pinitol treatment reversed the CsA-induced decline in the expression of Sirt1, increased phosphorylation of Akt and FoxO1, and acetylation of FoxO1. Although these are apparently different signalling pathways, D-pinitol administration appeared to ameliorate oxidative stress damage with subsequent reduction in fibrosis and apoptosis in chronic CsA-induced nephropathy.

$\mathrm{Nrf} 2$ is critical in cellular defense mechanism in response to increased oxidative stress (17). Upon oxidative insult, Nrf2 is dissociated from its cytoplasmic repressor Keap1, and then translocated to the nucleus, where it binds to antioxidant response element (ARE) to stimulate the transcription of its target genes, including HO-1, SOD1 and NQO1 $(17,21)$. Diverse mechanisms appear to be involved in the activation
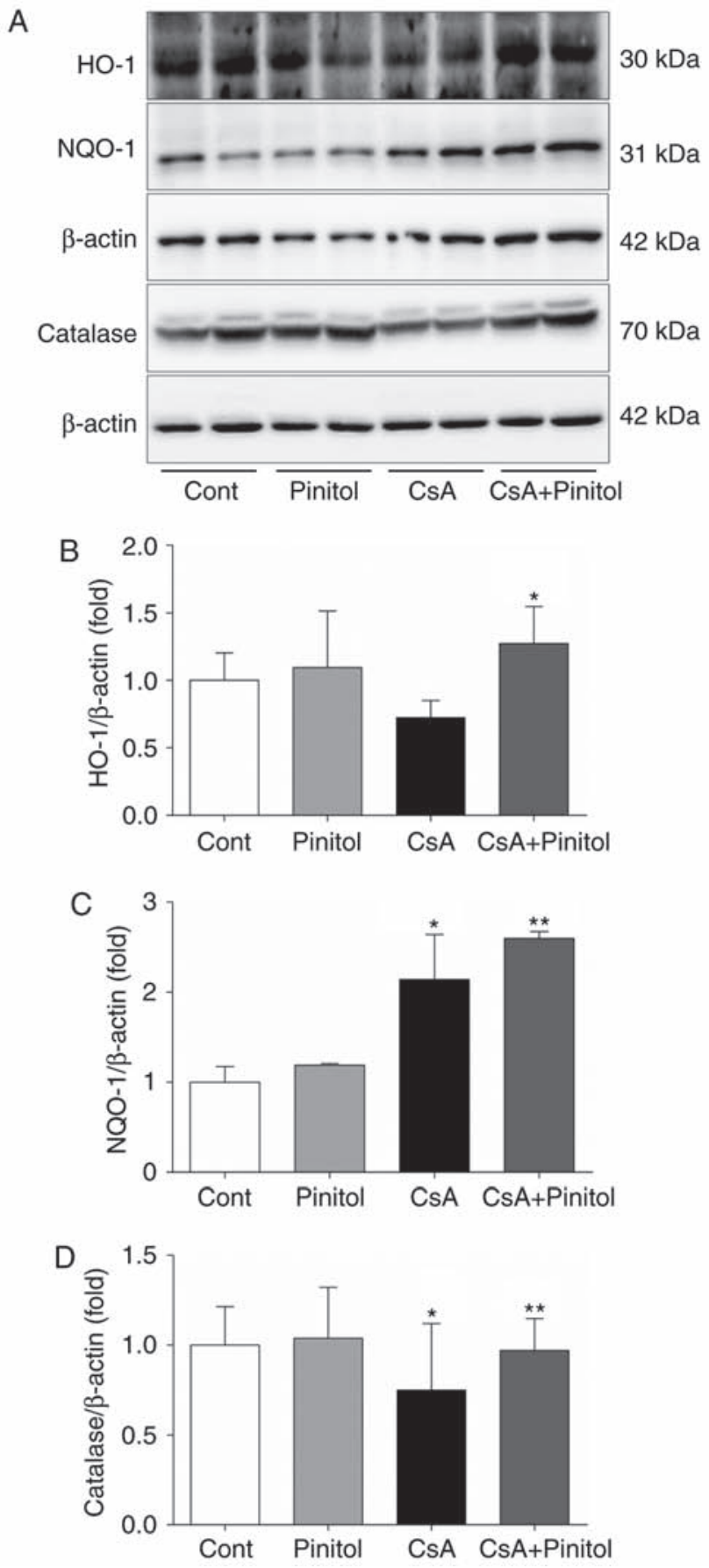

Figure 3. Effect of D-pinitol on renal expression of HO-1, NQO1 and catalase. (A) Representative western blot showing the effect of D-pinitol on the expression of HO-1, NQO1 and catalase in chronic CsA nephropathy. (B) Quantitative analyses of results for $\mathrm{HO}-1 / \beta$-actin. ${ }^{*} \mathrm{P}=0.002$ vs. CsA group. (C) Quantitative analyses of results for NQO1/ $\beta$-actin. ${ }^{*} \mathrm{P}<0.001$ vs. Cont group; ${ }^{* *} \mathrm{P}<0.001$ vs. Cont group. (D) Quantitative analyses of results for catalase $/ \beta$-actin. ${ }^{*} \mathrm{P}=0.016$ vs. Cont group; ${ }^{* *} \mathrm{P}=0.040$ vs. CsA group. Cont, vehicle-treated control group; Pinitol, D-pinitol; CsA, cyclosporine; HO-1, heme oxygenase-1; NQO1, NAD(P)H:quinone oxidoreductase 1.

of Nrf2, including the release of Nrf2 from Keap1, downregulated expression of Keap1, disruption of the Keap1-Cullin 3 complex, and the nuclear translocation of Nrf2 (21). Evidence supports that nuclear Nrf2 content is markedly declined with increased reactive oxygen species (ROS) production in chronic kidney disease $(15,22-24)$. Our previous studies also revealed a deterioration of the Nrf2 pathway with impairment 

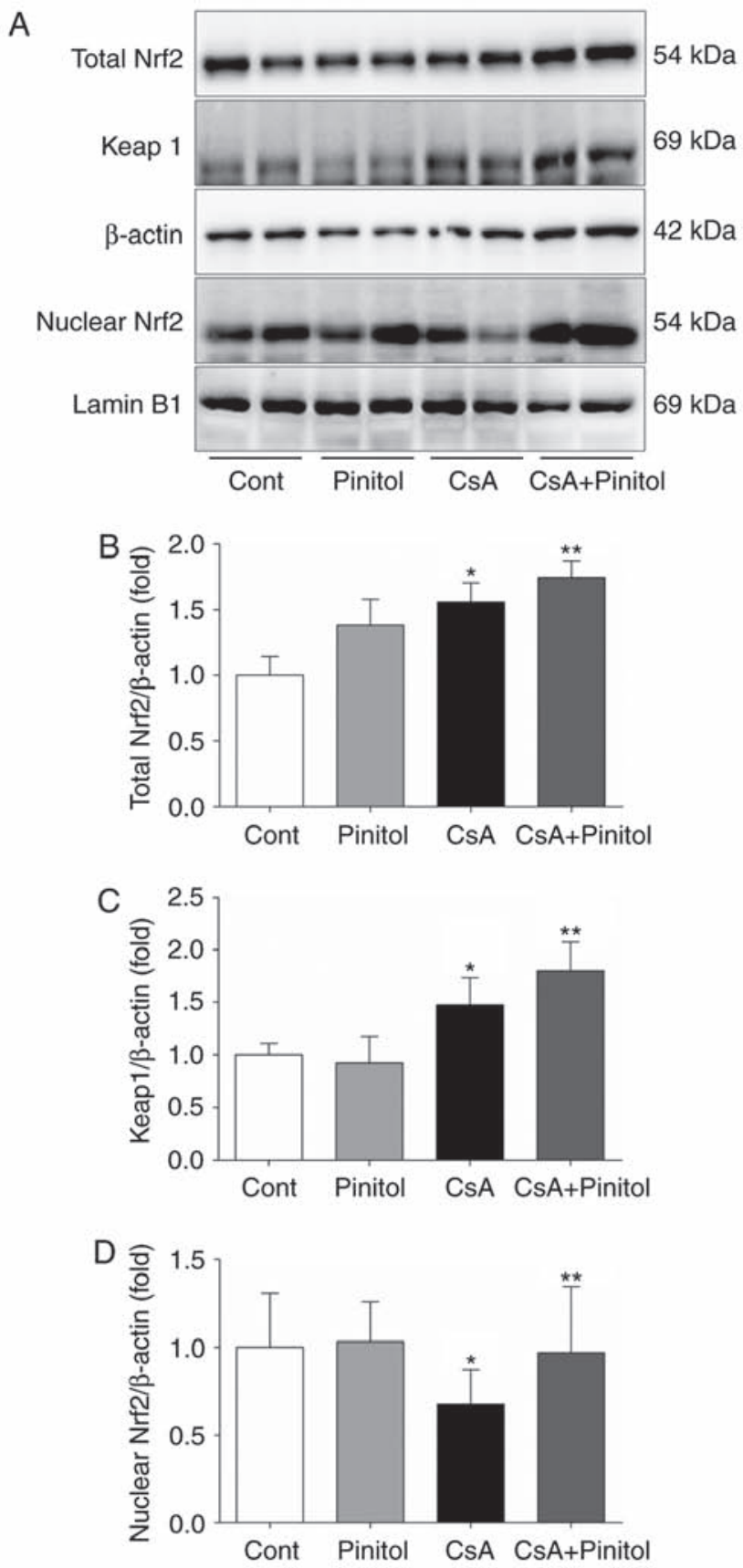

Figure 4. Effect of D-pinitol on renal expression of Nrf2, Keap 1 and nuclear Nrf2. (A) Representative western blot showing the effect of D-pinitol on the expression of Nrf2, Keap1 and nuclear Nrf2 in chronic CsA nephropathy. (B) Quantitative analyses of results for total Nrf2/ $\beta$-actin. ${ }^{*} \mathrm{P}=0.001 \mathrm{vs}$. Cont group; ${ }^{* *} \mathrm{P}<0.001$ vs. Cont group. (C) Quantitative analyses of results for Keap $1 / \beta$-actin. ${ }^{*} \mathrm{P}=0.010$ vs. Cont group. (D) Quantitative analyses of results for nuclear Nrf2/Lamin B1. $\mathrm{P}=0.008$ vs. Cont group; ${ }^{* *} \mathrm{P}=0.012$ vs. CsA group. Cont, vehicle-treated control group; Pinitol, D-pinitol; CsA, cyclosporine A; Nrf2, nuclear erythroid factor 2-related factor 2; Keap1, Kelch-like ech-associated protein 1 .

of downstream antioxidant defense mechanism in several experimental kidney disease models, including chronic CsA nephropathy $(17,20,25,26)$. In contrast to our previous study (17), the total renal expression of Nrf2 was increased by CsA treatment alone in the present study (Fig. 4A and B). Considering previous reports, which have shown various CsA concentrations in the blood $(23,27,28)$, this discrepancy may be due to differences in blood concentrations of CsA; the
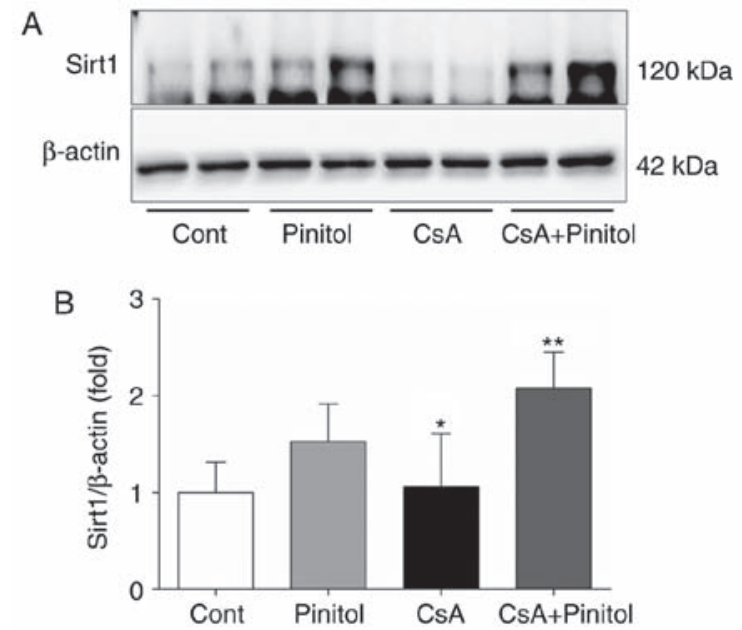

Figure 5. Effect of D-pinitol on renal expression of Sirt1 in chronic CsA nephropathy. (A) Representative western blot showing the effect of D-pinitol on the expression of Sirt1 in chronic CsA nephropathy. (B) Quantitative analyses for Sirt $1 / \beta$-actin. ${ }^{*} \mathrm{P}<0.001$ vs. Cont group; ${ }^{* *} \mathrm{P}=0.008$ vs. CsA group. Cont, vehicle-treated control group; Pinitol, D-pinitol; CsA, cyclosporine A; Sirt 1 , sirtuin 1 .

blood concentration of CsA was $\sim 1,800 \mathrm{ng} / \mathrm{ml}$ in our previous study (17) but was $<900 \mathrm{ng} / \mathrm{ml}$, almost half of the previous value, in the present study (Table I). It was observed that CsA provoked oxidative stress and the expression level of Nrf2 was induced by low level of ROS, but not by a high level of ROS (29). Therefore, although the mechanism involved in the discrepant effect of ROS on the expression of Nrf2 is in accordance with the concentration of ROS, it may be that lower blood concentrations of CsA increase the expression of Nrf2 via ROS. CsA also showed a discrepant effect on the expression of Nrf2. In the present study, the Nrf2 increased by CsA failed to stimulate the transcription of downstream antioxidant enzymes, which was incomplete and inadequate to protect the kidney from the nephrotoxicity caused by CsA itself (Figs. 1-4). It was hypothesized that this non-protective effect of Nrf2 on CsA-induced nephrotoxicity may be due to failure of the nuclear translocation of Nrf2 in CsA-treated mice (Fig. 4D). Based on these findings, CsA appeared to sequentially stimulate the expression of Nrf2, inhibit the nuclear translocation of Nrf2, and finally lose its effect on increasing the expression of $\mathrm{Nrf} 2$, as CsA concentration in the blood increased. However, D-pinitol treatment of the CsA-treated mice induced an increase in the nuclear expression of Nrf2 even with increased expression of Keap1, indicating that D-pinitol is a facilitator of the nuclear translocation of cytoplasmic Nrf2 released from Keap1. Taken together, D-pinitol appeared to attenuate CsA-induced renal injury through restoring the activity of Nrf2 and acting as a free radical scavenger $(9,30,31)$. It may protect the kidney against high levels of ROS by promoting the nuclear translocation of Nrf2 and scavenging low levels of ROS.

Another intracellular mechanism underpinning the effect of D-pinitol may be associated with the expression of Sirt1 coordinately with the Akt and FoxO1 pathway in the CsA nephropathy model. Sirtuin, an NAD-dependent deacetylase, has a renoprotective effect, and diverse target molecules through direct deacetylation or epigenetic gene modulation have been confirmed as effectors of its renoprotective 

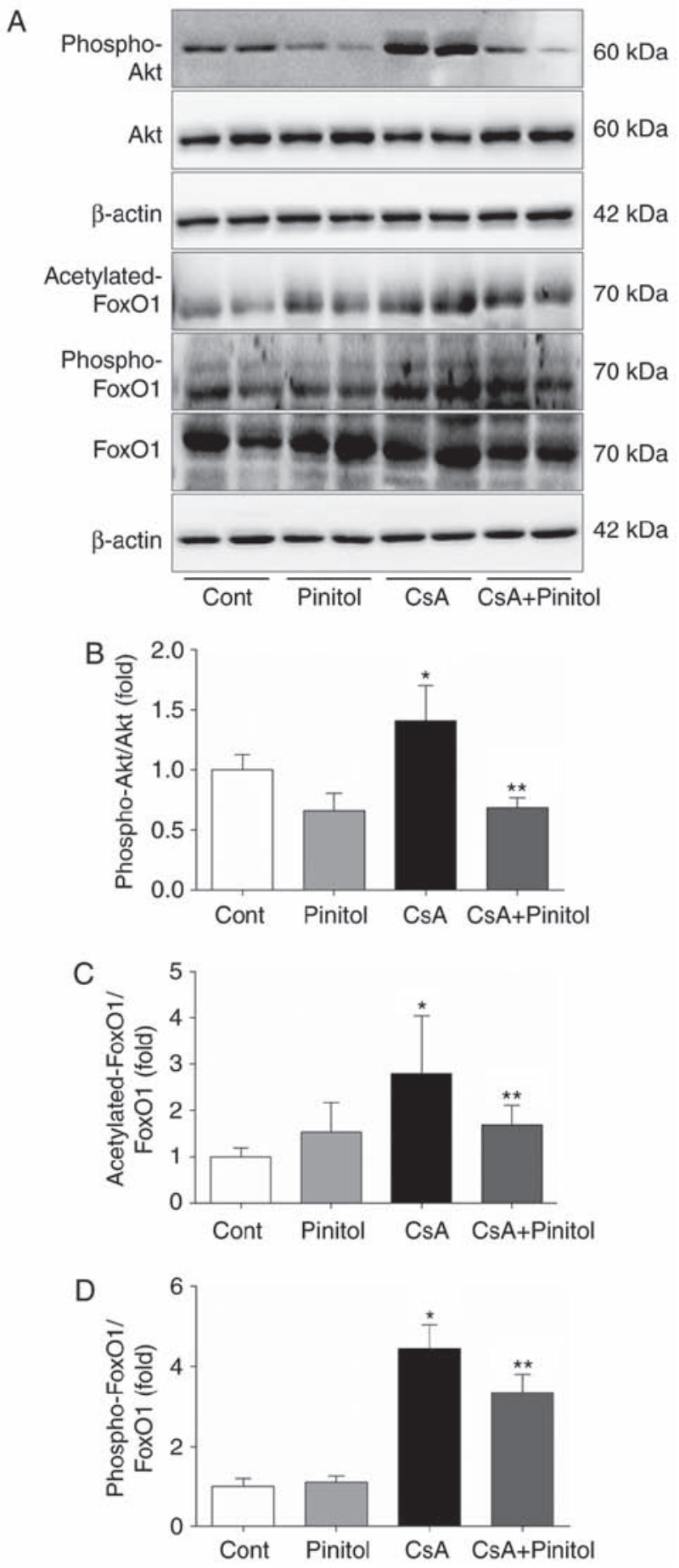

Figure 6. Effect of D-pinitol on renal expression of Akt and FoxO1 in chronic CsA nephropathy. (A) Representative western blot showing the effect of D-pinitol on the expression of phospho-Akt, Akt, acetylated FoxO1, phosphoFoxO1 and total FoxO1 in chronic CsA nephropathy. (B) Quantitative analyses for phospho-Akt/Akt. "P $<0.001$ vs. Cont group; ${ }^{* *} \mathrm{P}<0.001$ vs. CsA group. (C) Quantitative analyses for acetylated FoxO1/FoxO1. "P $<0.001$ vs. Cont group; ${ }^{* *} \mathrm{P}=0.013$ vs. CsA group. (D) Quantitative analyses for phospho-FoxO1/FoxO1. "P<0.001 vs. Cont group; ${ }^{* *} \mathrm{P}=0.012$ vs. CsA group. Cont, vehicle-treated control group; Pinitol, D-pinitol; CsA, cyclosporine A; FoxO1, Forkhead box O1; phospho, phosphorylated.

function (32). Among its isoforms, Sirtl has various substrates involved in energy metabolism with a crucial role in several biological pathways, including apoptosis, longevity and oxidative stress responses in chronic kidney diseases (33). The present study suggested a novel role of D-pinitol in restoring
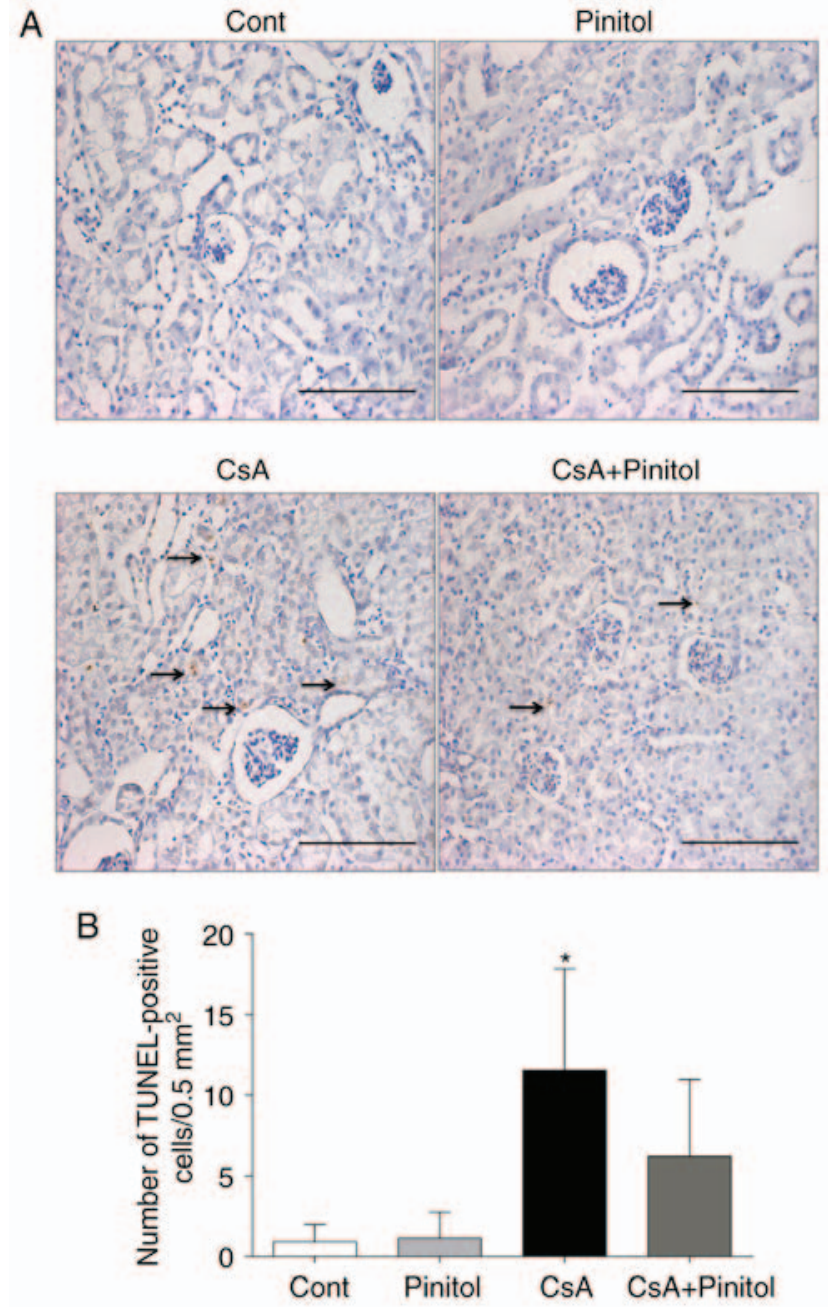

Figure 7. Effect of D-pinitol on apoptosis in chronic CsA nephropathy. (A) TUNEL assay in kidney tissues of experimental groups. Scale bar, $4 \mu \mathrm{m}$ (B) Quantitative analysis of TUNEL-positive nuclei in experimental groups. ${ }^{*} \mathrm{P}=0.002$ vs. CsA+pinitol group. Cont, vehicle-treated control group; Pinitol, D-pinitol; CsA, cyclosporine A; TUNEL, terminal deoxynucleotidyltransferase-mediated biotin nick end-labeling.

the altered expression of Sirt1 in a chronic CsA nephropathy model. As FoxO signalling can be selectively activated by Sirt1, the effect of D-pinitol on the expression of FoxO was investigated in the present study. The FoxO subfamily of Forkhead box transcription factors can activate an overlapping set of genes, which regulate cell cycle, apoptosis and metabolism, thereby coordinating cellular responses to various nutrient status and oxidative stress (34). Whereas nuclear Sirtl can deacetylate and reactivate the transcriptional activity of FoxO1, the inactivated form of FoxO1, which is acetylated by dissociation from Sirt1, can be translocated back to the cytoplasm (35). The present study showed that the expression of Sirt1 was decreased in the chronic CsA nephropathy model, whereas the ratio of acetylated FoxO1/FoxO1 was increased. Therefore, it may be that the suppression of Sirt1 by CsA caused increased acetylation of FoxO1, which then moved back to the cytoplasm with its ensuing inactivation. However, D-pinitol treatment reversed the changes of Sirt1 and FoxO1 to improve renal function and morphological derangement.

The activity of FoxO1 can be modulated by the PI3K/Akt pathway (34). The exposure of cells to oxidative stress can 
result in activation of the PI3K/Akt pathway and any fluctuation of Akt phosphorylation can be involved in the pathogenesis of several complex diseases (34). Akt allows the translocation of FoxO from the nucleus to the cytoplasm (34). Emerging evidence has shown that, upon oxidative stress, AKT is activated and subsequently phosphorylates FoxO1 proteins, which are then translocated to the cytoplasm (34-36). Modifications of FoxO1, including phosphorylation and acetylation, may assist in driving the expression of genes involved in combating oxidative stress $(2,36)$. It has been demonstrated that acetylated FoxO1 can become more sensitive to Akt-dependent phosphorylation, suggesting that acetylation and phosphorylation can cooperatively regulate the function of FoxO1 (37). To fine tune the activity of FoxO1 with complexity, this provides an additional dimension to the regulatory mechanism of FoxO1 associated with its dual post-translational modifications. The distinct modification of subsequent downstream FoxO1 by Sirtl and Akt may be involved in the renoprotective effect of D-pinitol in the CsA nephropathy model (38), although the mechanism at a molecular level requires further evaluation.

Although the results in the present study demonstrated a novel mechanism involved in the protective effect of D-pinitol against CsA-induced nephropathy, a number of points require addressing. First, unlike humans, mice are resistant to $\mathrm{CsA}$-induced renal injury due to $\mathrm{CsA}$-induced nephrotoxicity being species-specific. Therefore, salt depletion with higher-dose CsA is required to induce morphologic nephrotoxicity, compared with that in clinical practice (14). Secondly, the present study was unable to elucidate the molecular causality or the association between Nrf2 and Sirt1 in CsA-induced renal injury. Previous studies have suggested a plausible explanation. Kulkarni et al (39) showed that the fasting-induced activation of Sirt1 increased the expression of Nrf2 and accumulation of Nrf2 to the ARE region. It also activated ARE in tissues and cells of the liver, acting upstream of the Nrf2-ARE anti-oxidative pathway. Similarly, Huang et al (40) showed that the depletion of Sirt1 inhibited Nrf2-ARE pathway activation. It was concluded that Sirt1 regulation was a crucial promoter of the Nrf2-ARE pathway. However, how Sirt1 regulates the activity of Nrf2 or subsequent gene expression remains to be elucidated. Due to the response of Sirt1 to oxidative stress, the effect of Sirt1 on the Nrf2/ARE pathway may be determined and requires further investigation to elucidate key missing links. In conclusion, the present study showed that D-pinitol attenuated the CsA-induced renal fibrotic processes by mitigating oxidative stress through the Nrf2 and Sirt1-PI3K/Akt/FoxO1 pathways. This suggested that D-pinitol offers potential as a therapeutic agent for the progression of chronic tubulointerstitial fibrosis.

\section{Acknowledgements}

The authors would like to thank Dr Jong Hee Chung (Department of Statistics, The Graduate School of Ewha Womans University, Seoul, Republic of Korea) for her statistical advice. This study was supported by a grant (grant no. NRF-2015R1C1A1A02037258) of the Basic Science Research Program through the National Research Foundation of Korea funded by the Ministry of Science, ICT and Future Planning, Republic of Korea.

\section{Competing interests}

The authors declare there is no competing interest.

\section{References}

1. Lin TH, Tan TW, Tsai TH, Chen CC, Hsieh TF, Lee SS, Liu HH, Chen WC and Tang CH: D-pinitol inhibits prostate cancer metastasis through inhibition of alphaVbeta3 integrin by

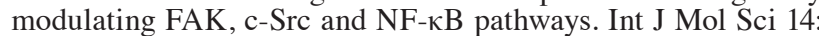
9790-9802, 2013.

2. Gao Y, Zhang M, Wu T, Xu M, Cai H and Zhang Z: Effects of D-pinitol on insulin resistance through the PI3K/Akt signaling pathway in type 2 diabetes mellitus rats. J Agric Food Chem 63: 6019-6026, 2015.

3. Choi MS, Lee MK, Jung UJ, Kim HJ, Do GM, Park YB and Jeon SM: Metabolic response of soy pinitol on lipid-lowering, antioxidant and hepatoprotective action in hamsters fed-high fat and high cholesterol diet. Mol Nutr Food Res 53: 751-759, 2009.

4. Sethi G, Ahn KS, Sung B and Aggarwal BB: Pinitol targets nuclear factor-kappaB activation pathway leading to inhibition of gene products associated with proliferation, apoptosis, invasion, and angiogenesis. Mol Cancer Ther 7: 1604-1614, 2008.

5. Rengarajan T, Nandakumar N, Rajendran P, Ganesh MK, Balasubramanian MP and Nishigaki I: D-pinitol mitigates tumor growth by modulating interleukins and hormones and induces apoptosis in rat breast carcinogenesis through inhibition of NF-kappaB. J Physiol Biochem 71: 191-204, 2015.

6. Dang NT, Mukai R, Yoshida K and Ashida H: D-pinitol and myo-inositol stimulate translocation of glucose transporter 4 in skeletal muscle of C57BL/6 mice. Biosci Biotechnol Biochem 74: 1062-1067, 2010

7. Hernández-Mijares A, Bañuls C, Peris JE, Monzó N, Jover A Bellod L, Victor VM and Rocha M: A single acute dose of pinitol from a naturally-occurring food ingredient decreases hyperglycaemia and circulating insulin levels in healthy subjects. Food Chem 141: 1267-1272, 2013.

8. Stull AJ, Wood KV, Thyfault JP and Campbell WW: Effects of acute pinitol supplementation on plasma pinitol concentration, whole body glucose tolerance, and activation of the skeletal muscle insulin receptor in older humans. Horm Metab Res 41: 381-386, 2009.

9. Sivakumar S, Palsamy P and Subramanian SP: Impact of D-pinitol on the attenuation of proinflammatory cytokines, hyperglycemia-mediated oxidative stress and protection of kidney tissue ultrastructure in streptozotocin-induced diabetic rats. Chem Biol Interact 188: 237-245, 2010.

10. Nankivell BJ, P'Ng CH, O'Connell PJ and Chapman JR: Calcineurin inhibitor nephrotoxicity through the lens of longitudinal histology: Comparison of cyclosporine and tacrolimus eras. Transplantation 100: 1723-1731, 2016.

11. Büscher AK, Beck BB, Melk A, Hoefele J, Kranz B, Bamborschke D, Baig S, Lange-Sperandio B, Jungraithmayr T, Weber LT, et al: Rapid response to cyclosporin A and favorable renal outcome in nongenetic versus genetic steroid-resistant nephrotic syndrome. Clin J Am Soc Nephrol 11: 245-253, 2016.

12. Gooch JL, King C,Francis CE, Garcia PS and Bai Y: Cyclosporine A alters expression of renal microRNAs: New insights into calcineurin inhibitor nephrotoxicity. PLoS One 12: e0175242, 2017.

13. Hošková L, Málek I, Kopkan Land Kautzner J: Pathophysiological mechanisms of calcineurin inhibitor-induced nephrotoxicity and arterial hypertension. Physiol Res 66: 167-180, 2017.

14. Lim SW, Doh KC, Jin L, Jin J, Piao SG, Heo SB, Chung BH and Yang CW: Ginseng treatment attenuates autophagic cell death in chronic cyclosporine nephropathy. Nephrology (Carlton) 19: 490-499, 2014

15. Ruiz S, Pergola PE, Zager RA and Vaziri ND: Targeting the transcription factor Nrf2 to ameliorate oxidative stress and inflammation in chronic kidney disease. Kidney Int 83: 1029-1241, 2013.

16. Yang CW, Ahn HJ, Kim WY, Shin MJ, Kim SK, Park JH, Kim YO, Kim YS, Kim J and Bang BK: Influence of the renin-angiotensin system on epidermal growth factor expression in normal and cyclosporine-treated rat kidney. Kidney Int 60: 847-857, 2001.

17. Hong YA, Lim JH, Kim MY, Kim EN, Koh ES, Shin SJ, Choi BS, Park CW, Chang YS and Chung S: Delayed treatment with oleanolic acid attenuates tubulointerstitial fibrosis in chronic cyclosporine nephropathy through $\mathrm{Nrf} 2 / \mathrm{HO}-1$ signaling. J Transl Med 12: 50, 2014. 
18. Reisman SA, Aleksunes LM and Klaassen CD: Oleanolic acid activates Nrf2 and protects from acetaminophen hepatotoxicity via Nrf2-dependent and Nrf2-independent processes. Biochem Pharmacol 77: 1273-1282, 2009.

19. Chung S, Kim S, Kim M, Koh ES, Yoon HE, Kim HS, Park CW, Chang YS and Shin SJ: T-type calcium channel blocker attenuates unilateral ureteral obstruction-induced renal interstitial fibrosis by activating the Nrf2 antioxidant pathway. Am J Transl Res 8: 4574-4585, 2016.

20. Kitada M and Koya D: Role of sirtuins in kidney disease. Curr Opin Nephrol Hypertens 23: 75-79, 2014.

21. Huang K, Huang J, Xie X, Wang S, Chen C, Shen X, Liu P and Huang H: Sirtl resists advanced glycation end products-induced expressions of fibronectin and TGF- $\beta 1$ by activating the Nrf2/ARE pathway in glomerular mesangial cells. Free Radic Biol Med 65: 528-540, 2013.

22. Parra Cid T, Conejo Garcia JR, Carballo Alvarez F and de Arriba G: Antioxidant nutrients protect against cyclosporine A nephrotoxicity. Toxicology 189: 99-111, 2003.

23. Yoon HE, Ghee JY, Piao S, Song JH, Han DH, Kim S, Ohashi N, Kobori H, Kuro-o M and Yang CW: Angiotensin II blockade upregulates the expression of klotho, the anti-ageing gene, in an experimental model of chronic cyclosporine nephropathy. Nephrol Dial Transplant 26: 800-813, 2011.

24. Kim HJ and Vaziri ND: Contribution of impaired Nrf2-Keap1 pathway to oxidative stress and inflammation in chronic renal failure. Am J Physiol Renal Physiol 298: F662-F671, 2010.

25. Chung S, Yoon HE, Kim SJ, Kim SJ, Koh ES, Hong YA, Park CW, Chang YS and Shin SJ: Oleanolic acid attenuates renal fibrosis in mice with unilateral ureteral obstruction via facilitating nuclear translocation of Nrf2. Nutr Metab (Lond) 11: 2, 2014.

26. Kim S, Kim SJ, Yoon HE, Chung S, Choi BS, Park CW and Shin SJ: Fimasartan, a novel angiotensin-receptor blocker, protects against renal inflammation and fibrosis in mice with unilateral ureteral obstruction: The possible role of nrf2. Int J Med Sci 12: 891-904, 2015

27. Piao SG, Kang SH, Lim SW, Chung BH, Doh KC, Heo SB, Jin L, $\mathrm{Li} \mathrm{C}$ and Yang CW: Influence of $\mathrm{N}$-acetylcysteine on klotho expression and its signaling pathway in experimental model of chronic cyclosporine nephropathy in mice. Transplantation 96 : 146-153, 2013.

28. Doh KC, Lim SW, Piao SG, Jin L, Heo SB, Zheng YF, Bae SK, Hwang GH, Min KI, Chung BH and Yang CW: Ginseng treatment attenuates chronic cyclosporine nephropathy via reducing oxidative stress in an experimental mouse model. Am J Nephrol 37: 421-433, 2013.

29. Gęgotek A and Skrzydlewska E: The role of transcription factor Nrf2 in skin cells metabolism. Arch Dermatol Res 307: 385-396, 2015.
30. Sivakumar S, Palsamy P and Subramanian SP: Attenuation of oxidative stress and alteration of hepatic tissue ultrastructure by $\mathrm{D}$-pinitol in streptozotocin-induced diabetic rats. Free Radic Res 44: 668-678, 2010.

31. Kim MJ, Yoo KH, Kim JH, Seo YT, Ha BW, Kho JH, Shin YG and Chung $\mathrm{CH}$ : Effect of pinitol on glucose metabolism and adipocytokines in uncontrolled type 2 diabetes. Diabetes Res Clin Pract 77 (Suppl 1): S247-S251, 2007.

32. Carafa V, Rotili D, Forgione M, Cuomo F, Serretiello E, Hailu GS, Jarho E, Lahtela-Kakkonen M, Mai A and Altucci L: Sirtuin functions and modulation: From chemistry to the clinic. Clin Epigenetics 8: 61, 2016.

33. Wakino $\mathrm{S}$, Hasegawa $\mathrm{K}$ and Itoh $\mathrm{H}$ : Sirtuin and metabolic kidney disease. Kidney Int 88: 691-698, 2015.

34. Medema RH and Jäättelä M: Cytosolic FoxO1: Alive and killing. Nat Cell Biol 12: 642-643, 2010.

35. Zhao Y, Yang J, Liao W, Liu X, Zhang H, Wang S, Wang D, Feng J, Yu L and Zhu WG: Cytosolic FoxO1 is essential for the induction of autophagy and tumour suppressor activity. Nat Cell Biol 12: 665-675, 2010.

36. Szydłowski M, Jabłońska E and Juszczyński P: FOXO1 transcription factor: A critical effector of the PI3K-AKT axis in B-cell development. Int Rev Immunol 33: 146-157, 2014.

37. Matsuzaki H, Daitoku H, Hatta M, Aoyama H, Yoshimochi K and Fukamizu A: Acetylation of Foxol alters its DNA-binding ability and sensitivity to phosphorylation. Proc Natl Acad Sci USA 102: 11278-11283, 2005.

38. Kim DH, Park CH, Park D, Choi YJ, Park MH, Chung KW, Kim SR, Lee JS and Chung HY: Ginsenoside Rc modulates Akt/FoxO1 pathways and suppresses oxidative stress. Arch Pharm Res 37: 813-820, 2014.

39. Kulkarni SR, Donepudi AC, Xu J, Wei W, Cheng QC, Driscoll MV, Johnson DA, Johnson JA, Li X and Slitt AL: Fasting induces nuclear factor E2-related factor 2 and ATP-binding cassette transporters via protein kinase A and sirtuin-1 in mouse and human. Antioxid Redox Signal 20: 15-30, 2014.

40. Huang K, Chen C, Hao J, Huang J, Wang S, Liu P and Huang H: Polydatin promotes Nrf2-ARE anti-oxidative pathway through activating Sirt1 to resist AGEs-induced upregulation of fibronetin and transforming growth factor- $\beta 1$ in rat glomerular messangial cells. Mol Cell Endocrinol 399: 178-189, 2015.

This work is licensed under a Creative Commons Attribution-NonCommercial-NoDerivatives 4.0 International (CC BY-NC-ND 4.0) License. 